\title{
O Que é, Afinal, Marketing de Relacionamento? Uma Proposta de Conceito Unificador
}

\section{Fernanda Bueno Cardoso Scussel}

Doutoranda em Administração pelo Programa de Pós-Graduação em Administração da Universidade Federal de Santa Catarina (PPGAdm/UFSC). Florianópolis, Sc. Brasil.E-mail: fbcardoso@gmail.com

\section{Martin de la Martinière Petroll}

Professor do Programa de Pós-Graduação em Administração e do Departamento de Administração da Universidade Federal de Santa Catarina. Florianópolis, Sc. Brasil. E-mail: martin_petroll@hotmail.com

\section{Elder Semprebon}

Professor do Departamento de Administração da Universidade Federal do Paraná. Curitiba, PR. Brasil. E-mail: elder.semprebon@gmail.com

\section{Rudimar Antunes Da Rocha}

Professor do Programa de Pós-Graduação em Administração e do Departamento de Administração da Universidade Federal de Santa Catarina. Florianópolis, Sc. Brasil. E-mail: rrudimar@hotmail.com

\section{Resumo}

Segundo Grönroos (2009), Agariya e Singh (2011), Demo e Rozzett (2013) e Demo et al. (2015), acredita-se que ainda não há consenso sobre a definição de marketing de relacionamento, os autores entendem que seja necessária uma limpeza conceitual a partir do fomento de um conceito unificador. Sendo assim, o presente estudo teórico atualiza a pesquisa de Agaryia e Singh (2011) a partir de uma revisão sistemática das publicações com base em um levantamento bibliométrico sobre o tema entre os anos de 2011 e 2015. Foram identificados e analisados 69 artigos, categorizados segundo critérios demográficos, institucionais e metodológicos. Partindo dos principais assuntos debatidos sobre marketing de relacionamento, foram identificados elementos definidores e, assim, propôs-se um conceito unificador. Como principal contribuição, este artigo apresenta um conceito novo, baseado na literatura clássica de marketing de relacionamento associada às mais recentes perspectivas na área, de forma a entregar uma definição capaz de auxiliar o desenvolvimento teórico-empírico do conhecimento em marketing de relacionamento.

Palavras-chave: Marketing de Relacionamento. Colaboradores Internos. Parceiros. Clientes. Valor.

\section{Abstrat}

According to Grönroos (2009), Agariya and Singh (2011), Demo and Rozzett (2013) and Demo et al. (2015), there is still no consensus on a definition to relationship marketing, the reason why the authors understand that a conceptual cleaning is necessary, demanding an unifying concept. Therefore, the present theoretical study updates the research of Agaryia and Singh (2011), with a systematic review of the publications based on a bibliometric survey on the theme between 2011 and 2015. A total of 69 articles were identified and analyzed, categorized according to demographic, institutional and methodological criteria. Starting from the main issues discussed on relationship marketing, defining elements were identified and, thus, an unifying concept was proposed. As a main contribution, this article presents a new concept for the construct, based on the classic relationship marketing literature associated to the latest perspectives in the field, in order to provide a definition capable of assisting the theoretical-empirical development of knowledge in relationship marketing.

Keywords: Relationship Marketing. Employees. Partners. Clients. Value. 


\section{INTRODUÇÃO}

O marketing de relacionamento surgiu na década de 1980 e rapidamente tornou-se foco de interesse de pesquisadores e profissionais de marketing, motivados pelo aumento da complexidade e incerteza dos comportamentos dos mercados e pela introdução $e$ sofisticação das tecnologias de informação, as quais permitiram trocas eficazes entre as empresas e também coletas de dados sobre o comportamento do consumidor em escala global (BALLANTYNE; CHRISTOPHER; PAYNE, 2003).

Sendo assim, entende-se que o marketing de relacionamento consista na atração, manutenção $e$ desenvolvimento de relacionamentos com clientes (BERRY, 1983). Portanto, a sua contribuição está atrelada ao monitoramento e identificação de oportunidades no mercado $e$, ainda, ao estabelecimento de relacionamentos duradouros com clientes e parceiros de negócios (DEMO et al., 2015).

Contudo, apesar da visibilidade e da popularidade que o termo marketing de relacionamento conquistou em mais de 30 anos, o debate acerca de seu real significado ainda é considerado uma importante lacuna na literatura. Isso porque o vocábulo marketing de relacionamento foi utilizado para expressar diferentes significados, como taxa de retenção de clientes, programas de fidelidade, comportamento de compra repetitivo, estratégia empresarial e, onde reside o maior equívoco, sinônimo para CRM (Customer Relationship Management) ou Gestão de Relacionamento com o Cliente (BALLANTYNE; CHRISTOPHER; PAYNE, 2003; FROW; PAYNE, 2009; GRÖNROOS, 2009; DEMO et al., 2015).

Discute-se, assim, que a ausência de consenso sobre a definição de marketing de relacionamento pode ter como consequências a incompreensão de seus objetivos e a negligência dos seus pressupostos e benefícios (ZABLAH; BELLENGER; JOHNSTON, 2004; GRÖNROOS, 2009; AGARIYA; SINGH, 2011; DEMO et al., 2015). Para Payne (2006), essa discussão torna-se relevante devido à significativa influência que a maneira como o marketing de relacionamento é entendido, implementado e praticado nas organizações afeta os resultados organizacionais.

Todavia, ainda que seja latente a necessidade de limpeza conceitual, poucos são os estudos voltados à construção de conceitos e identificação de construtos definidores, privilegiando estudos teórico-empíricos, o que reforça a carência de debates capazes de fomentar um conceito unificador (GRÖNROOS, 2009; AGARIYA; SINGH, 2011; DEMO; ROZZETT, 2013; DEMO et al., 2015).

Agaryia e Singh (2011), por exemplo, conduziram uma extensa bibliometria abarcando os períodos entre 1982 e 2010 com o intuito de identificar os conceitos propostos para marketing de relacionamento e seus principais construtos. O estudo encontrou 72 conceitos e 146 construtos definidores para marketing de relacionamento. Mas entende-se que a proposição de um conceito único para o marketing de relacionamento capaz de abarcar seus pressupostos, objetivos e consequências seja possível e necessário, contribuindo, assim, para a literatura de marketing e para a aplicação gerencial do marketing de relacionamento.

Nesse sentido, o presente estudo realizou uma revisão bibliométrica da produção nacional e internacional sobre marketing de relacionamento entre 2011 e 2015, seguindo e atualizando a pesquisa de Agaryia e Singh (2011) com o objetivo de identificar as bases constitutivas do marketing de relacionamento e de propor um conceito unificador.

\section{Referencial Teórico}

O marketing de relacionamento se origina dos estudos sobre marketing de serviços, nos quais a ênfase se desloca para relacionamentos, tendo como marco histórico o artigo seminal de Berry (1983), para quem o marketing de relacionamento consiste em atrair, manter e desenvolver relacionamentos com clientes. Para o autor, a conquista de um cliente é apenas uma parte do processo, sendo que solidificar o relacionamento, transformar clientes indiferentes em clientes leais e tornar consumidores em clientes deve ser considerado marketing. Na mesma perspectiva, Vavra (1993) argumenta que o relacionamento entre consumidor e empresa tem início com a venda e é construído a partir da interação continuada, designada pós-venda.

Nesse contexto, o marketing tradicional, com base nas transações, torna-se insuficiente para explicar a nova realidade. Adota-se, então, o foco nos relacionamentos como nova perspectiva, surgindo o 
marketing de relacionamento como paradigma alternativo, visando envolver e integrar consumidores às atividades de marketing, onde o relacionamento de caráter integrador sugere uma aproximação econômica, emocional e estrutural entre as partes, tendo como consequência relacionamentos duradouros e lucrativos (SHETH; PARVATIYAR, 1995).

Durante essa transição, porém, o entendimento do significado de marketing de relacionamento ficou comprometido. De acordo com Grönroos (2009), o marketing de relacionamento pressupõe a atividade de gerenciamento dos relacionamentos com os consumidores, ainda que a literatura compreenda distintas noções do que isto significa. O termo é facilmente identificado como sinônimo de retenção de clientes, banco de dados e programas de fidelidade (D'ANGELO; SCHNEIDER; LARÁN, 2006). Ryals (2005) atenta para a utilização do vocábulo como taxa de retenção de clientes, explicando que sua utilização puramente como estratégia lucrativa pode elevar os custos da empresa, impedindo os possíveis benefícios do marketing de relacionamento. Grönroos (2009) acrescenta, ainda, que o entendimento sobre o construto muitas vezes se limita a uma situação de ganha-ganha entre consumidor e empresa, à criação de comprometimento mútuo ou ao comportamento de compra repetitivo.

Dada a confusão conceitual, O'Malley e Tynan (2000) avaliam que essa dissonância acarreta a negligência de aspectos essenciais do marketing de relacionamento, como a orientação a longo prazo, o desenvolvimento de vínculos emocionais e a confiança.

Ademais, Sheth e Parvatiyar (1995) defendem que o marketing de relacionamento não se limita a uma estratégia competitiva, mas sim a uma filosofia integradora, onde os clientes são envolvidos às atividades de marketing por meio da aproximação econômica, emocional e estrutural entre eles e as empresas. Berry e Gresham (1986) preconizam que se trata de um processo que passa por aprendizagem, gerenciamento $e$ atendimento das preferências dos clientes, e cujo resultado será a criação de vantagens e ofertas customizadas para os clientes e lealdade para a organização.

Conforme Vavra (1993), o marketing de relacionamento abarca sete atividades fundamentais à criação e utilização das informações dos clientes, sendo elas: (1) a preparação e organização das informações obtidas sobre os clientes em um banco de dados; (2) a programação de pontos de contatos com os clientes; (3) a análise das interações com os clientes e transformação dos dados em informações; (4) a elaboração de indicadores de satisfação; (5) o desenvolvimento de programas de comunicação com os clientes; (6) o patrocínio de eventos ou programações especiais para os clientes; e (7) a identificação e recuperação de clientes perdidos.

Diante da importância do relacionamento com clientes e da necessidade de seu gerenciamento, surge, nos anos 1990, um modelo de gestão denominado Customer Relationship Management (CRM), ou Gestão de Relacionamento com o Cliente (DEMO; ROZZETT, 2013). Para Bretzke (2000), o CRM consiste na integração do marketing e da tecnologia da informação, com o objetivo de fornecer às organizações meios eficazes e integrados de atender, reconhecer e cuidar dos clientes. Na visão de Payne (2006), o CRM prevê o desenvolvimento de relacionamentos com os clientes por meio da união de ferramentas da tecnologia da informação (TI) e das estratégias e pressupostos do marketing de relacionamento.

Sendo assim, a falta de convergência a respeito de uma definição para marketing de relacionamento somada ao surgimento do CRM e da similaridade dos conceitos e objetivos fez com que, muitas vezes, os construtos fossem utilizados como sinônimos (DEMO et al., 2015). Ainda que comum na literatura, trata-se de um equívoco, visto que, segundo Zablah, Bellenger e Johnston (2004), o marketing de relacionamento é o precursor filosófico do CRM. Ballantyne, Christopher e Payne (2003) concordam que o marketing de relacionamento propicia o surgimento de outros subtemas, entre eles o CRM. Na mesma linha, Frow e Payne (2009) asseguram que o marketing de relacionamento consiste no gerenciamento estratégico de todos os relacionamentos estabelecidos entre as firmas e os stakeholders, enquanto o CRM compreende o gerenciamento estratégico dos relacionamentos com os clientes, com apropriação da tecnologia da informação. Logo, tem-se o marketing de relacionamento como um construto principal do qual o CRM faz parte.

Destarte, torna-se relevante diferenciar e conceituar os termos. Do lado do marketing de relacionamento, Sheth e Partatiyar (1995) reforçam que o consenso sobre sua real definição permitirá a operacionalização do conceito que, por sua vez, permitirá que organiza- 
ções ajam de forma conjunta para entregar valor ao cliente. A depuração do conceito também impactará nos estudos em CRM, já que, para Payne (2006), a busca de uma limpeza conceitual para CRM não se limita a um preciosismo semântico. Para o autor, o CRM utiliza as estratégias de marketing de relacionamento combinadas ao potencial da tecnologia da informação, visando relacionamentos lucrativos de longo prazo. É razoável dizer, a partir da literatura visitada, que o emprego do CRM sempre utiliza os pressupostos do marketing de relacionamento, mas nem todas as estratégias de marketing de relacionamento precisam se valer da tecnologia da informação para serem eficientes. Confirma-se, assim, que a clareza nas definições tende a auxiliar estudos na linha de marketing de relacionamento e CRM.

No que tange às discussões sobre marketing de relacionamento, Healy et al. (2001) consideram que o construto abrange uma concepção ampla, devendo ser entendido como um conceito "guarda-chuva". Frow e Payne (2009) assinalam que essa noção de amplitude faz com que possam ser identificadas definições distintas e muitas vezes confusas a respeito do que realmente significa marketing de relacionamento. Conforme Grönroos (2009), o termo marketing de relacionamento tem sido usado para indicar uma gama de estratégias, como o comportamento de compra repetitivo, programas de fidelidade, conquista de novos clientes e até mesmo como uma ferramenta de marketing utilizada para manipular consumidores. Considerando o exposto, observa-se que a definição para o construto é vital para o avanço dos estudos na área.

Os benefícios consequentes do marketing de relacionamento também despertam forte interesse dos pesquisadores, seja acerca do desenvolvimento de novos produtos (ERNST et al., 2011), na capacidade inovativa das empresas (BATTOR; BATTOR, 2010; LIN; CHEN; CHIU, 2010) ou, ainda, sobre o crescimento dos níveis de confiança, satisfação e lealdade (ABBADE, 2014). Por exemplo, no que tange à lealdade, registram-se pesquisas sobre fidelização de clientes e pós-venda (MENDES; PEDRON, 2014; COMIOTTO et al., 2014; AMARAL et al., 2015), sobre confiança (KAUR; SHARMA; MAHAJAN, 2012; MILAN; DE TONI, 2012; SOARES; BORTOLUZZO; BARROS, 2012), benefícios relacionais (ROCHA; CLARO, 2013;
LEE; KIM; PAN, 2014; MOTA, 2014; HUANG, 2015), engajamento entre empresas e clientes (ASHLEY et al., 2011; HANSLIN; RINDELL, 2014), e qualidade do relacionamento (AA; BLOEMER; HENSELER, 2013), em que os temas supracitados encontram-se associados à construção dos relacionamentos verdadeiros $e$ duradouros.

O marketing de relacionamento é associado, ainda, a estudos sobre desempenho empresarial, sugerindo a criação de uma cultura baseada no valor para o consumidor e no gerenciamento global dos relacionamentos (LING-YEE, 2011). Aqui, tem-se o aparecimento do marketing interno como elemento-chave à operacionalização da orientação para o consumidor (WU; TSAI; FU, 2013; LOURENÇO; SETTE, 2013). Sob tal perspectiva, surgem estudos que analisam a relação entre marketing de relacionamento e cultura organizacional (MAGALHÃES; CRNKOVIC; MORETTI, 2010; IGLESIAS; SAUQUET; MONTAÑA, 2011; CREMONEZI et al., 2013; LOURENÇO, 2014).

Em relação às revisões de literatura sobre marketing de relacionamento, o estudo conduzido por Das (2009) apresentou o panorama da pesquisa em marketing de relacionamento entre 1994 e 2006. O autor identificou 209 artigos relacionados ao tema marketing de relacionamento, cuja fonte era composta por 56 periódicos pertencentes a bases de dados distintas. O resultado revelou também que a literatura em marketing de relacionamento pode ser classificada em cinco categorias, a saber: (1) objetivos, abarcando satisfação do cliente, retenção e lealdade; (2) construtos que definem marketing de relacionamento, como confiança, compromisso, cooperação e qualidade do relacionamento; (3) instrumentos, como marketing direto, banco de dados, marketing um-para-um; (4) implicações, tais como privacidade, impacto da cultura, influência da tecnologia; e (5) aplicação gerencial, onde se inserem os programas de implementação $e$ as práticas industriais.

Já Agaryia e Singh (2011) conduziram uma extensa bibliometria no período entre 1982 e 2010 para identificar os conceitos propostos para marketing de relacionamento e seus principais construtos. Foram identificadas 72 definições para marketing de relacionamento, sendo que o cerne de todas elas envolve aquisição, retenção, aumento do lucro, orientação para longo prazo e uma situação de ganha-ganha para os 
envolvidos no relacionamento. Identificaram, ainda, 146 construtos definidores, sendo os mais citados a confiança, a satisfação/experiência, a lealdade, o comprometimento, a qualidade do serviço e a comunicação. Por fim, Agaryia e Singh (2011) encontraram que os setores nos quais o marketing de relacionamento é mais explorado são o bancário, o de seguros e o de saúde.

A revisão de literatura feita por Agaryia e Singh (2011) revelou que a pesquisa em marketing de relacionamento pode ser classificada em cinco categorias, a saber: (1) objetivos; quais seriam os resultados esperados com a implementação do marketing de relacionamento, especialmente no tocante à satisfação e retenção de clientes; (2) construtos definidores que conduzem ao marketing de relacionamento, como confiança, comprometimento, cooperação e proximidade; (3) instrumentos, como marketing direto, marketing de serviços e CRM; (4) obstáculos, como os demográficos - cultura, gênero, idade - e os tecnológicos; e (5) aplicação gerencial, definida em termos da implementação dos programas e das práticas da indústria.

Faria et al. (2014) analisaram a produção acadêmica em marketing de relacionamento durante vinte anos (de 1992 a 2012), revelando que a produção brasileira em CRM compreendeu 96 artigos, com destaque para a predominância de pesquisas B2C (business-to-consumer) voltadas ao consumidor final. Por fim, o trabalho publicado por Demo et al. (2015), a partir da análise da produção nacional no período entre 2000 e 2013 revelou a existência de 52 artigos indicando a predominância de trabalhos teórico-empíricos, especialmente voltados para o mercado consumidor.

Os resultados supracitados reforçam a relevância e o vigor da pesquisa em marketing de relacionamento. Não obstante, o necessário desenvolvimento teórico sobre o tema é escasso, sendo privilegiados os estudos teórico-empíricos, reforçando a carência de debates capazes de fomentar um conceito unificador (GRÖNROOS, 2009; AGARIYA; SINGH, 2011; DEMO; ROZZETT, 2013; DEMO et al., 2015).

Em relação à definição para marketing de relacionamento, a literatura revela-se ainda bastante pulverizada. De acordo com Berry (1983), precursor do tema, o marketing de relacionamento pode ser conceituado como os esforços para atração, manutenção e desenvolvimento de relacionamentos com clientes.
Para Morgan e Hunt (1994), o marketing de relacionamento consiste em todas as atividades de marketing orientadas para o estabelecimento, desenvolvimento e manutenção de trocas relacionais bem-sucedidas. Sheth e Partatiyar (1995) relatam que o marketing de relacionamento é uma teoria integradora, cujo escopo contempla canais de distribuição, marketing de serviços, comportamento do consumidor, pesquisa de marketing, comunicação e estratégia. As definições registradas mostram-se pouco compreensíveis ou operacionalizáveis devido à amplitude do seu escopo e da ausência de convergência entre os construtos definidores (AGARIYA; SINGH, 2011).

Para Agariya e Singh (2011), apesar do esforço feito por grandes pensadores em marketing, a investigação realizada não encontrou uma definição adequada para marketing de relacionamento, tampouco os autores propuseram um conceito para ele. Cabe registrar que trabalhos recentemente publicados reforçam a pertinência de uma definição apropriada e unificadora (DEMO; PESSOA, 2015; DEMO; BATELLI; ALBUQUERQUE, 2015; DEMO et al., 2015).

Destarte, o quadro conceitual para marketing de relacionamento revela o vigor da pesquisa, bem como a relevância de suas implicações gerenciais. Atesta, ainda, o excesso de definições para marketing de relacionamento, a consequente ausência de um conceito unificador e a necessidade de tal conceito. Assim, visto que o estudo de Agaryia e Singh (2011) abarcou os estudos publicados até 2010 e diversos pesquisadores citam a evolução veloz e transformadora do marketing de relacionamento nos últimos anos, tem-se reforçada a necessidade da atualização de conceitos e pilares desse construto, somando-se, ainda, a identificação de lacunas de pesquisa na temática.

\section{Método}

O procedimento metodológico empregado neste artigo foi o de desk research, envolvendo um conjunto de artigos sobre marketing de relacionamento publicados entre os anos de 2011 e 2015. Esse recorte justifica-se por ser uma atualização do que propuseram Agariya e Singh (2011), responsáveis pelo último grande levantamento concernente ao marketing de relacionamento. Considerou-se apenas artigos de peri- 
ódicos acadêmicos, uma vez que configuram pesquisas mais consolidadas. Quanto às bases de dados, foram utilizadas as seguintes: Scielo, EBSCO, Emerald Full Text, ScienceDirect, Elsevier, Sage e Springer. Foram selecionados os artigos que contivessem as palavras-chave "marketing de relacionamento", ou "relationship marketing".

A seleção dos artigos foi feita por meio da Systematic Review, ou revisão sistemática, proposta por Dybå e Dingsøyr (2008), que consiste em um método estruturado para identificar estudos relevantes sobre determinado tema. Nessa metodologia, em cada estágio da pesquisa extraem-se os trabalhos selecionados, que em seguida são submetidos à nova revisão. $\mathrm{O}$ processo de filtragens sucessivas deste trabalho, constituindo protocolo de pesquisa, segundo os autores supramencionados, é apresentado na Figura 1.

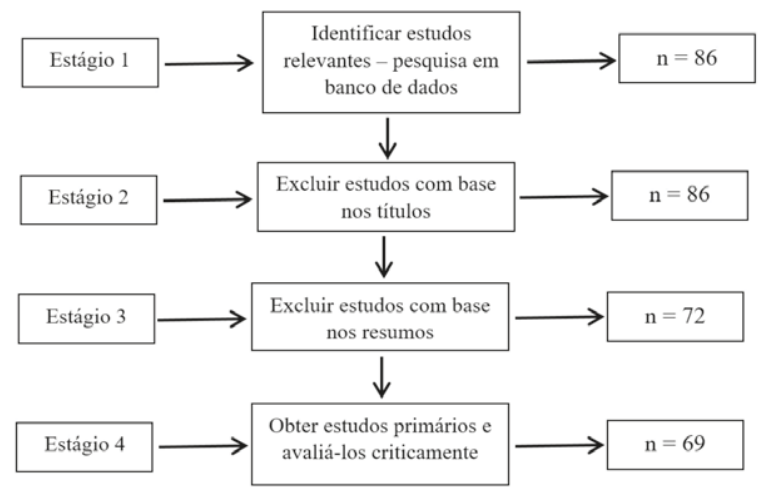

Figura 1: Protocolo da seleção de estudos Fonte: Adaptado de Dybå e Dingsøyr (2008)

O primeiro estágio resultou um total de 86 trabalhos, utilizando-se como parâmetro de busca as palavras-chave, sendo a base para o processo de seleção dos artigos, assim como fizeram Agariya $e$ Singh (2011). Na sequência, Dybå e Dingsøyr (2008) recomendam a exclusão de artigos duplicados ou publicados em duas fontes e que não condisseram com a temática em questão, estágio em que nenhum trabalho foi excluído. Em seguida, o estágio de leitura dos resumos resultou em 72 trabalhos. Por fim, os trabalhos foram submetidos à leitura crítica do seu inteiro teor, considerando-se aqueles que tratam mais diretamente do tema da pesquisa e não apenas o citam. Restaram, dessa forma, 69 artigos que atenderam às especificidades do protocolo de pesquisa.

Selecionadas as fontes de coleta de informações, estipularam-se quais informações deveriam ser extra- ídas dos artigos: o(s) nome(s) do(s) autor(es), país e instituição de origem, ano de publicação, o enquadramento dos estudos (teóricos ou teórico-empíricos), os autores que mais publicaram no período, o ramo de atuação explorado pelas pesquisas e as categorias estipuladas por Agaryia e Singh (2011) no que se refere aos objetivos, aos construtos definidores de marketing de relacionamento, aos seus instrumentos, obstáculos e à sua aplicação gerencial.

\section{Apresentação e Discussão dos Resultados}

Esta seção compila, em primeira instância, a síntese ilustrada do levantamento dos 69 artigos identificados na literatura de marketing nos últimos cinco anos. Com base nesses resultados, foi elaborado $e$ descrito o cenário da produção científica sobre marketing de relacionamento, seguido das discussões acerca das definições sobre o tema, convidando a reflexões e engendrando sugestões para futuras pesquisas.

\subsection{Análise da Produção Científica sobre Marketing de Relacionamento entre 2011 e 2015}

O período analisado foi produtivo em relação ao tema, haja vista que foram 69 publicações em cinco anos, uma média de 13 artigos por ano, conforme ilustrado no Gráfico 1.

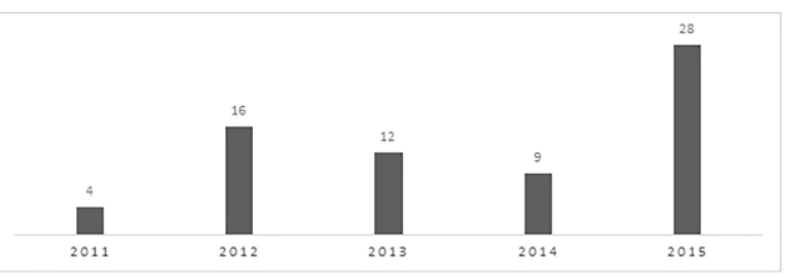

Gráfico 1: Artigos sobre marketing de relacionamento publicados entre 2011 e 2015.

Fonte: Elaborado pelos autores deste artigo

Observa-se que o marketing de relacionamento se manteve em evidência nos últimos cinco anos, com um crescimento vertiginoso em 2015. O fato de ainda haver publicações sobre marketing de relacionamento décadas após o seu surgimento pode ser atribuído à utilização do marketing de relacionamento como fonte de vantagem competitiva sustentável, à sua cada vez 
maior popularização e ao crescimento do número de grupos de pesquisa que estudam marketing de relacionamento (AGARIYA; SINGH, 2011; DEMO et al., 2015). Os fatores supracitados, com o respaldo do número considerável observado de publicações, reforçam a literatura quando esta alerta para a urgência de um conceito unificador de marketing de relacionamento.

A maior parte dos estudos analisados enquadrou-se no modelo teórico-empírico, com $78 \%$ das publicações, sendo apenas $22 \%$ focadas em discussões teóricas. Esses resultados corroboram o postulado por Agariya e Singh (2011), ao afirmarem que os estudos teórico-empíricos superam, em volume de publicação, as discussões teóricas no campo do marketing de relacionamento.

Dentre os 69 artigos encontrados, alguns autores obtiveram destaque ao publicarem mais de um artigo dentro do período contemplado pelo estudo. O Quadro 1 apresenta o nome desses autores, suas instituições e país de origem $e$ o total de artigos publicados por cada um.

\begin{tabular}{|c|c|c|c|}
\hline Autor & $\begin{array}{l}\text { INSTITUIÇÃO DE } \\
\text { ORIGEM }\end{array}$ & País & $\begin{array}{c}\text { Número dE } \\
\text { Artigos }\end{array}$ \\
\hline $\begin{array}{l}\text { Arun Kumar } \\
\text { Agariya }\end{array}$ & $\begin{array}{l}\text { Indian Institute of } \\
\text { Information Technology } \\
\& \text { Management }\end{array}$ & Índia & 4 \\
\hline Gisela Demo & $\begin{array}{l}\text { Universidade } \\
\text { de Brasília }\end{array}$ & Brasil & 4 \\
\hline $\begin{array}{l}\text { Cristiane } \\
\text { Drebes Pedron }\end{array}$ & $\begin{array}{l}\text { Universidade } \\
\text { Nove de Julho }\end{array}$ & Brasil & 3 \\
\hline Deepali Singh & $\begin{array}{l}\text { Indian Institute of } \\
\text { Information Technology } \\
\text { \& Management }\end{array}$ & Índia & 3 \\
\hline $\begin{array}{c}\text { Cléria Donizete } \\
\text { da Silva } \\
\text { Lourenço }\end{array}$ & $\begin{array}{c}\text { Universidade } \\
\text { Federal de Lavras }\end{array}$ & Brasil & 2 \\
\hline Hamid Tohidi & Islamic Azad University & Irã & 2 \\
\hline $\begin{array}{l}\text { Mark } \\
\text { Tadajewski }\end{array}$ & Durham University & $\begin{array}{l}\text { Reino } \\
\text { Unido }\end{array}$ & 2 \\
\hline $\begin{array}{l}\text { Mohammad } \\
\text { Mehdi Jabbari }\end{array}$ & Islamic Azad University & Irã & 2 \\
\hline $\begin{array}{l}\text { Nadia Kassouf } \\
\text { Pizzinatto }\end{array}$ & Unimep & Brasil & 2 \\
\hline $\begin{array}{l}\text { Antonio Carlos } \\
\text { Giuliani }\end{array}$ & $\begin{array}{c}\text { Universidade Metodista } \\
\text { de Piracicaba }\end{array}$ & Brasil & 2 \\
\hline
\end{tabular}

Quadro 1: Autores que mais publicaram artigos sobre marketing de relacionamento entre 2011 e 2015

Fonte: Elaborado pelos autores deste artigo
No que tange aos assuntos mais estudados, há a predominância do tema aplicação gerencial, onde estão inseridas as investigações acerca da implementação dos programas de marketing de relacionamento $e$ as práticas das empresas. Em segundo lugar, tem-se a categoria objetivos, que compreende os estudos voltados aos resultados esperados com o marketing de relacionamento. Na sequência está a categoria instrumentos, na qual constam as ferramentas utilizadas dentro do marketing de relacionamento, compreendendo, também, os trabalhos voltados ao desenvolvimento de escalas de mensuração do construto. A categoria construtos definidores encontra-se em seguida, abarcando as discussões sobre os pilares constitutivos do marketing de relacionamento. Nenhum dos artigos destina-se à discussão sobre obstáculos enfrentados pelo marketing de relacionamento, revelando importantes lacunas na literatura, já que Agariya e Singh (2011) apontam a necessidade de explorar fatores demográficos e tecnológicos no contexto relacional. Esses resultados encontram-se ilustrados no Gráfico 2.

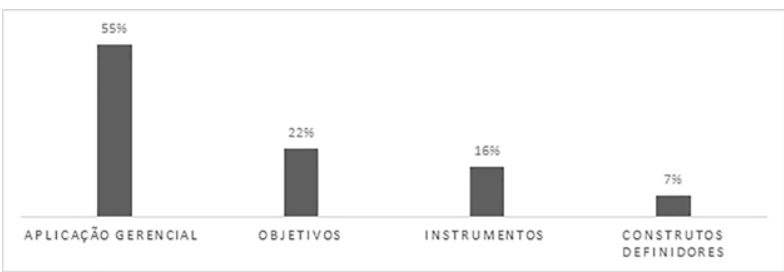

Gráfico 2: Assuntos mais estudados em marketing de relacionamento entre 2011 e 2015.

Fonte: Elaborado pelos autores deste artigo

Com base no exposto, observa-se a ocorrência de estudos concentrados na análise de fenômenos específicos, observados a partir da prática gerencial e da aplicação do marketing de relacionamento nos negócios. A observada predominância de estudos teórico-empíricos confirma a relevância do tema, mas enseja o desenvolvimento teórico, especialmente no que se refere à construção de definições que permitiria uma melhoria na operacionalização dos conceitos. Verificou-se, ainda, o crescente esforço no desenvolvimento e validação de instrumentos de pesquisa capazes de mensurar construtos dentro do marketing de relacionamento (DEMO; ROZZETT, 2013; DEMO; PESSOA, 2015; DEMO; BATTELLI; ALBUQUERQUE, 2015). A criação de instrumentos válidos contribui para a produção científica na área de marketing, sejam eles de natureza qualitativa ou quantitativa, visto que per- 
mitem a mensuração de construtos e o entendimento da percepção dos consumidores e da realidade das organizações. Ademais, a validação de instrumentos de marketing de relacionamento também viabilizará outros estudos relacionais nas áreas de marketing $e$ comportamento do consumidor.

Ainda, Agariya e Singh (2011) concordam que o levantamento do ramo de atuação das empresas pesquisadas nos trabalhos teórico-empíricos é fundamental ao mapeamento do que está sendo investigado em marketing de relacionamento. Demo et al. (2015) acrescentam que essa etapa auxilia a saber onde está o foco das pesquisas, bem como a identificação de lacunas na literatura.

Dos 69 estudos selecionados, 54 são artigos teórico-empíricos e abarcam 37 diferentes ramos de atuação. Dada a pulverização desses setores, o Gráfico 3 apresenta os ramos de atuação mais expressivos, sendo aqueles que figuraram em pelo menos dois trabalhos.

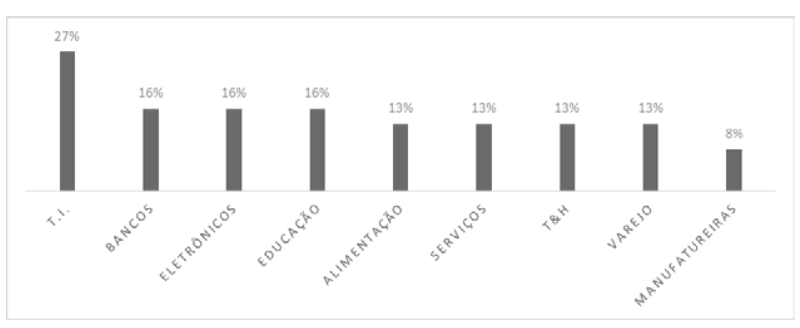

Gráfico 3: Ramo de atuação das empresas pesquisadas entre 2011 e 2015.

Fonte: Elaborado pelos autores deste artigo

As empresas participantes desses estudos concentram-se na área da tecnologia da informação, presente em $27 \%$ das publicações, resultado que talvez possa ser reflexo da confusão conceitual sobre os termos marketing de relacionamento e CRM se for considerado o conteúdo dessas publicações levantadas pelo presente estudo, fortalecendo, assim, a imprescindibilidade de uma limpeza conceitual (FROW; PAYNE, 2009; ASHLEY et al., 2011). Na sequência, encontram-se as empresas do setor bancário, de eletrônicos e educacional, presentes, cada uma, em $16 \%$ dos artigos. A seguir, com $13 \%$ cada, estão as empresas de alimentação, serviços, turismo e hotelaria e varejo e, com $8 \%$, as empresas manufatureiras.

Esses resultados corroboram a relevância do mercado consumidor (DEMO; ROZZETT, 2013), embora em suas origens o marketing de relacionamento tenha tido um enfoque em canais de distribuição, dentro das indústrias de manufatura (GRÖNROOS, 2009). Ademais, as investigações sobre o setor de serviços corroboram a literatura quando expõe que serviços e relacionamentos estão interligados (BERRY, 1983; VARGO; LUSCH, 2008; GRÖNROOS, 2009). Por fim, a presença, ainda que modesta, das empresas manufatureiras tem respaldo nos estudos sobre canais de distribuição e relacionamentos no mercado corporativo (WILSON; VLOVSKY, 1997).

\subsection{Marketing de Relacionamento e suas Definições}

No tocante às definições para marketing de relacionamento utilizadas pelos autores do período compreendido entre 2011 e 2015, é notória a pulverização dos esforços em conceituar o construto. Dentre os 69 artigos pesquisados, três autores tiveram destaque, visto que suas definições foram alvo de citação em 28 dos artigos encontrados: Berry (1983), Morgan e Hunt (1994) e Payne (2006). A frequência com que os autores são citados e as respectivas definições encontram-se no Quadro 2.

\begin{tabular}{|c|c|c|}
\hline DEFINIÇÃo & Autor & $\begin{array}{l}\text { NúMERo de } \\
\text { CitaÇõEs } \\
\text { Literais }\end{array}$ \\
\hline $\begin{array}{l}\text { Marketing de relacionamento } \\
\text { consiste em todas as atividades } \\
\text { de marketing orientadas } \\
\text { para o estabelecimento, } \\
\text { desenvolvimento e } \\
\text { manutenção de trocas } \\
\text { relacionais bem-sucedidas. }\end{array}$ & $\begin{array}{c}\text { Morgan e } \\
\text { Hunt (1994) }\end{array}$ & 13 \\
\hline $\begin{array}{l}\text { Abordagem estratégica } \\
\text { holística para gerenciar } \\
\text { o relacionamento com } \\
\text { clientes, a fim de criar } \\
\text { valor para o acionista. }\end{array}$ & Payne (2006) & 8 \\
\hline $\begin{array}{l}\text { Atração, retenção } \\
\text { e manutenção de } \\
\text { relacionamentos com } \\
\text { os consumidores. }\end{array}$ & Berry (1983) & 7 \\
\hline
\end{tabular}

Quadro 2: Definições para marketing de relacionamento Fonte: Elaborado pelos autores deste artigo

Todavia, é preciso salientar que Payne (2006) não é um autor clássico de marketing de relacionamento, mas um notório autor na área de CRM, utilizando os 
pressupostos do marketing de relacionamento aliados à tecnologia da informação em nome da criação de inteligência de mercado para as empresas e, consequentemente, resultados mais competitivos.

As definições supracitadas, ainda que norteiem boa parte das publicações em marketing de relacionamento, podem ser questionadas acerca de sua compatibilidade com a atual situação de competição vivida pelas organizações. Para Grönroos (2009), falta às definições encontradas na literatura a forma como o processo de marketing acontece, que deve ir além da simples noção de estabelecimento, manutenção $e$ desenvolvimento de relacionamentos.

Quanto aos artigos de Berry (1983) e Morgan e Hunt (1994), verificou-se a convergência entre as definições utilizadas. Não obstante, cabe registrar que esses autores não propuseram conceitos para marketing de relacionamento, mas apresentaram apenas definições operacionais nos contextos dos fenômenos observados. Esses conceitos são válidos, contudo, espera-se que a definição do construto marketing de relacionamento seja unificadora, ou seja, aplicável a qualquer contexto, e, principalmente, oriunda de um desenvolvimento teórico. As definições operacionais propostas em Berry (1983) e Morgan e Hunt (1994), presentes nos artigos analisados, convergiram dentro dos temas apresentados no Quadro 3.

\begin{tabular}{|c|c|}
\hline TeMAS DAS DEFINIÇÕES OPERACIONAIS & $\begin{array}{c}\text { NÚMERO DE } \\
\text { CITAÇõES }\end{array}$ \\
\hline $\begin{array}{c}\text { Marketing de relacionamento como criação } \\
\text { de relacionamentos de longo prazo }\end{array}$ & 10 \\
\hline Marketing de relacionamento como estratégia & 7 \\
\hline $\begin{array}{c}\text { Marketing de relacionamento como } \\
\text { gerenciamento da interação entre } \\
\text { empresa, clientes e atividades }\end{array}$ & 3 \\
\hline $\begin{array}{c}\text { Marketing de relacionamento como } \\
\text { sinônimo de retenção de clientes }\end{array}$ & 2 \\
\hline $\begin{array}{c}\text { Marketing de relacionamento como } \\
\text { ferramenta para criação de valor }\end{array}$ & 2 \\
\hline $\begin{array}{c}\text { Marketing de relacionamento como } \\
\text { transações contínuas e frequentes entre } \\
\text { compradores e fornecedores }\end{array}$ & 2 \\
\hline
\end{tabular}

Quadro 3: Definições operacionais para marketing de relacionamento

Fonte: Elaborado pelos autores deste artigo
Com base nos temas que auxiliaram as definições operacionais, verifica-se que o marketing de relacionamento ainda é utilizado como sinônimo de estratégia e retenção de clientes ou, ainda, como uma ferramenta de marketing (RYALS, 2005). Observa-se, também, que mesmo com a evolução das pesquisas em marketing de relacionamento e os benefícios obtidos com a sua aplicação gerencial, o construto ainda é visto apenas como trocas frequentes, contrariando o postulado pela literatura a respeito da mudança do paradigma de trocas para o paradigma relacional (SHETH; PARVATIYAR, 1995). Ainda, Pupovac, Zehetner e Sudarevic (2012) apontam que, apesar da popularização do marketing de relacionamento, as empresas ainda não utilizam as informações dos clientes da forma mais adequada, associando isto à falta de esclarecimento sobre definição e propósitos.

Os resultados aqui descritos corroboram os achados de Agariya e Singh (2011), ao afirmarem que não há consenso na literatura em relação a um conceito unificador. Ademais, a operacionalização de um conceito deve ser posterior à sua definição, bem como as hipóteses devem ser teoricamente justificadas, comprometendo os resultados (KASSARJIAN, 1971). Assim, no intuito de identificar quais os elementos essenciais à construção de uma definição para marketing de relacionamento, os artigos utilizados para esta bibliometria foram lidos na íntegra, tendo sido reconhecidos 59 construtos definidores, analisados por sua relevância, considerando a citação em pelo menos cinco dos artigos avaliados. O Quadro 4 apresenta esses achados.

\begin{tabular}{|c|c|}
\hline Construtos Definidores & Número de Citações \\
\hline Confiança & 20 \\
\hline Satisfação & 19 \\
\hline Lealdade & 15 \\
\hline Cocriação & 13 \\
\hline Qualidade do Relacionamento & 9 \\
\hline Comprometimento & 8 \\
\hline Comunicação & 7 \\
\hline Marketing Interno & 7 \\
\hline Valor & 7 \\
\hline Benefícios Relacionais & 6 \\
\hline Fidelização & 6 \\
\hline
\end{tabular}




\begin{tabular}{|c|c|}
\hline Gestão do Conhecimento & 6 \\
\hline Cultura Organizacional & 5 \\
\hline Engajamento & 5 \\
\hline Experiência & 5 \\
\hline Interação & 5 \\
\hline
\end{tabular}

Quadro 4: Construtos Definidores

Fonte: Elaborado pelos autores deste artigo

Comparando os resultados por ora obtidos com os apresentados por Agariya e Singh (2011), observa-se a importância da confiança, satisfação e lealdade como pilares para o marketing de relacionamento. Ambos os estudos trazem os construtos definidores supracitados no topo de suas listas. Encontram-se nas duas relações, ainda, os construtos comprometimento, comunicação, valor, fidelização, gestão do conhecimento e qualidade do relacionamento. Há de se ressaltar, contudo, que Agariya e Singh (2011) apresentam satisfação e experiência como sinônimos, resultado que não foi replicado na presente análise, na qual os artigos selecionados tratam satisfação e experiência como construtos definidores distintos.

Os resultados aqui tecidos revelam que novos construtos definidores passam a fazer parte das bases formadoras do marketing de relacionamento no atual cenário. Benefícios relacionais, cocriação, engajamento, interação, cultura organizacional e marketing interno passam a integrar, dessa forma, o entendimento sobre marketing de relacionamento.

Os construtos definidores engajamento e benefícios relacionais voltam-se para a decisão do consumidor em assumir ou não um relacionamento com as empresas. Identifica-se, assim, um esforço das empresas no sentido de demonstrar benefícios capazes de atrair o consumidor em um contexto relacional, deixando para ele a decisão do relacionamento. Essa noção encontra embasamento na literatura, no momento em que Grönroos (2009) defende que os consumidores podem estar em modo relacional ou não relacional. Para o autor, ainda que o gerenciamento de consumidores como relacionamentos seja eficiente, não se pode relacionar automaticamente um cliente a uma empresa.

Sobre cocriação, engajamento e interação, Grönroos (2009) observa que, qualquer que seja a definição para marketing de relacionamento, ela não pode excluir a perspectiva da criação de valor para o consumidor, visto que os resultados do marketing de relacionamento estão atrelados à criação de valor percebido para os clientes por meio do tempo. Nesse contexto, Srinivasan et al. (2002) relatam que a vantagem-chave das interações entre firma e consumidor são as oportunidades de a empresa se engajar com os processos dos consumidores. Nessas interações, os processos e as atividades dos consumidores se tornam expostos à empresa, permitindo que a empresa direcione seus esforços de forma que possa influenciar a criação de valor que ali acontece (GRÖNROOS, 2009).

No que tange à incorporação dos construtos definidores cultura organizacional e marketing interno ao escopo do conceito de marketing de relacionamento, esta encontra respaldo na literatura, quando se pressupõe o marketing de relacionamento como um processo que permeie toda a empresa (SILVA et al., 2012; LOURENÇO, 2014; SEMPREBON et al., 2014). Nesse sentido, a literatura sugere a necessidade de expandir o foco no consumidor para além da área de marketing, atrelando o marketing de relacionamento à necessidade de uma cultura organizacional compatível com seus propósitos, objetivos e resultados (GRÖNROOS, 2009).

Na mesma linha, Agariya e Yayi (2015) sugerem que a compreensão dos conceitos de marketing de relacionamento tem impacto não somente nas relações entre clientes e empresas e entre firmas, mas impacta também no marketing interno e na forma como os funcionários replicam com os clientes externos o tratamento por eles recebido e como isso pode refletir nos resultados organizacionais. Além do mais, Grönroos (2009) advoga que a criação de valor está relacionada à atividade da empresa como um todo.

Cabe ressaltar que esses construtos foram identificados em estudos teóricos e em pesquisas empíricas realizadas em diferentes setores da economia, países distintos e contextos culturais diversos. Dessa forma, a convergência dos construtos definidores forma uma estrutura capaz de orientar a construção de um conceito unificador, cuja aplicabilidade seja generalizável.

A análise dos 69 artigos selecionados revela a ampliação do escopo de marketing de relacionamento, originalmente centrado na relação com consumidores externos. Visto que se trata de uma área do conhecimento atrelada à prática e aos movimentos dos mercados e das sociedades, o marketing de relacionamento 
passa a considerar outras perspectivas, considerando o relacionamento com outros atores e outras formas de se operacionalizar dentro das empresas. Tem-se, então, a necessidade da atualização dos conceitos clássicos. A literatura apoia tais interpretações ao apontar que a importância de uma definição correta vai além de um preciosismo semântico, visto que afeta de forma significativa os impactos de como o construto é compreendido, implementado e praticado nas organizações (PAYNE, 2006; RICHARDS; JONES, 2008).

Posto isso, sugere-se, como conceito unificador, que o marketing de relacionamento consiste em uma filosofia de negócio que promove a interação de colaboradores internos, parceiros e clientes visando o engajamento com a organização $e$ criação de valor para todas as partes.

Esse conceito abrange os novos construtos definidores, abarcando as perspectivas atuais no que tange aos estudos sobre marketing de relacionamento, conforme sugerem ser necessário Agayia e Singh (2011). Convém assinalar que o ponto central desse conceito reside na palavra interação. $\mathrm{O}$ sentido que se busca para o termo, dentro do conceito aqui construído, converge com o que Alderson (1957) considera sobre valor: o valor criado quando os produtos são usados é mais importante do que os valores de troca, tanto para a organização quanto para os clientes. Nesse prisma, o real valor de troca só se concretiza se existir criação de valor para o consumidor, o que só pode ocorrer por meio da interação (BALLANTYNE; VAREY, 2006; GRÖNROOS, 2007, 2008). Destarte, são as interações que permitem a transformação do valor potencial em valor real para o consumidor (GRÖNROOS, 2008).

No que tange às partes envolvidas na interação, faz-se mister esclarecer que a criação de valor está na esfera dos consumidores, ou seja, as empresas não criam valor, oferecendo apenas valor em potencial, que são concretizados apenas na percepção dos consumidores (GRÖNROOS, 2008, 2009). Os consumidores adquirem os bens, os implementam em suas atividades diárias e, a partir de então, transformam o valor potencial em valor real (GRÖNROOS, 2008). Para o autor, compete às empresas oferecerem os recursos aos consumidores - seus produtos e serviços - e estabelecerem interações com os indivíduos enquanto eles aplicam esses recursos, a fim de cocriar valor junto aos consumidores. Essa cocriação de valor traduz-se, no conceito proposto, como engajamento. Recorrendo à literatura, isso se torna possível por meio da compreensão das necessidades, hábitos e estilo de vida dos consumidores e seus processos geradores de valor, que permitirá o desenvolvimento de novos produtos e serviços (GRÖNROOS, 2008; 2009).

No que tange aos colaboradores, esses estão integrados ao conceito proposto haja vista que a literatura preconiza o elemento recursos humanos como peça chave no desenvolvimento das interações (FREITAS; CUNICO; PEDRON, 2015). Conforme os autores, o alinhamento dos colaboradores com a estratégia de construção de relacionamentos com clientes acontece por meio da construção de habilidades e competências, somada à remuneração e à comunicação transparente. Verifica-se, então, a aderência do conceito proposto à abordagem holística defendida por Grönroos (2009). Corroboram, ainda, Demo et al. (2015), ao postularem que o marketing de relacionamento deve ser encarado como filosofia empresarial. Nesse sentido, ampliou-se a perspectiva para "filosofia de negócio" para que seja possível que o marketing de relacionamento esteja presente onde houver qualquer transação comercial, não se limitando à atividade empresarial, mas a todo negócio que se beneficie do desenvolvimento de relacionamentos.

Como norte para o desenvolvimento do conceito, recorreu-se a Grönroos (2009), quando esse autor assegura que uma definição completa de marketing de relacionamento deve compreender a identificação, estabelecimento, manutenção e desenvolvimento de relacionamentos, mas também englobar perspectivas mais modernas, não abarcadas pelos autores clássicos, auxiliando acadêmicos e profissionais de negócios a compreenderem e implementarem a estratégia relacional nos mercados corporativos e nos mercados consumidores. Destarte, o conceito aqui proposto revela-se coerente com o que pregavam os autores clássicos (BERRY, 1983; 1995), uma vez que defende o desenvolvimento de relacionamentos duradouros com clientes; adequado ao cenário organizacional, visto que tem raízes em estudos teórico-empíricos recentes, considerando a realidade de cada empresa, indústria e contexto, de forma a provocar benefícios a todos os que fornecem estímulos e sofrem impacto à atividade organizacional, ampliando o escopo de uma relação ganha-ganha ou, ainda, da simples transação comercial. 


\section{Considerações Finais}

O presente estudo almejou, a partir de uma revisão sistemática da literatura e por meio de um levantamento bibliométrico da produção sobre marketing de relacionamento no período compreendido entre os anos de 2011 e 2015, sanar ou pelo menos mostrar um caminho de consenso sobre o conceito de marketing de relacionamento. Como consequência, buscou-se realizar uma limpeza conceitual com o estabelecimento de um conceito unificador de marketing de relacionamento (GRÖNROOS, 2009; AGARIYA; SINGH, 2011; DEMO; ROZZETT, 2013; DEMO et al., 2015). Como principal resultado, foi apresentado um conceito unificador para marketing de relacionamento, coerente com os pressupostos indicados pela literatura da área e com a necessidade atual das organizações e dos consumidores.

No que tange às implicações do conceito, este extrapola os limites do departamento de marketing, tornando as estratégias de marketing de relacionamento perpassem toda a organização, envolvendo todos os colaboradores. Ademais, com base no que se abordou sobre interação e criação de valor, o conceito proposto preconiza o envolvimento das organizações com os consumidores durante a utilização dos produtos, cabendo a estas o desenvolvimento de ações que permitam o engajamento e os benefícios mútuos.

No tocante às limitações, vale assinalar que não se pretendeu esgotar a literatura concernente ao tema no período investigado em razão de se ter restringido as buscas às palavras-chave marketing de relacionamento ou relationship marketing e a bases de dados acessíveis aos autores do presente estudo. Além disso, trata-se de um estudo teórico, o que pressupõe a ausência de operacionalização e validação empírica do conceito unificador aqui estabelecido.

Posto isso, em relação às futuras investigações sobre marketing de relacionamento, primeiramente recomenda-se a verificação empírica do conceito unificador proposto em diferentes ramos de atuação organizacional e, ainda, em diferentes contextos. Aconselha-se fortemente explorar a relação entre marketing de relacionamento e recursos humanos, haja vista a relevância dos colaboradores para a consecução das estratégias de relacionamento com os clientes. Nesse sentido, estudos que relacionem o marketing de relacionamento com cultura organizacional são fundamentais para o desenvolvimento da teoria na área. Além desses caminhos, vislumbra-se a possibilidade e a relevância de se estudar marketing de relacionamento e o contexto cultural, ou seja, na perspectiva de como as trocas de valor ocorrem em diferentes regiões. Por fim, tendo esclarecido o que é marketing de relacionamento, considera-se salutar ao conhecimento teórico-empírico da temática a relação com outras variáveis de marketing e do contexto organizacional como um todo, considerando a perspectiva unificadora aqui defendida.

\section{REFERÊNCIAS}

AA, Z. V.; BLOEMER, J.; HENSELER, Jörg. Using customer contact centres as relationship marketing instruments. Service Business, [S.I.], v. 9, n. 2, p. 185208, 2013.

ABBADE, E. B. A Percepção de Clientes Universitários sobre o Relacionamento com suas Operadoras de Telefonia Móvel. REGE Revista de Gestão, [S.l.], v. 21, n. 3, p. 433-448, 2014.

AGARIYA, A. K.; SINGH, D. What really defines relationship marketing? A review of definitions and general and sector-specific defining constructs. Journal of Relationship Marketing, [S.I.], v. 10, n. 4, p. 203-237, 2011.

AGARIYA, A. K.; YAYI, S. H. ERM Scale Development and Validation in Indian IT Sector. Journal of Internet

Banking and Commerce, [S..l], v. 20, n. 1, 2015.

\section{ALDERSON, W. Marketing behavior and executive}

action. Homwood, IL: Richard D. Irwin (Inc.), 1957.

AMARAL, T. C. et al. Influências do Comércio Eletrônico B2B no Processo de Atendimento de Pós-Vendas no Segmento de Bens de Capital: Estudo de Caso em uma Empresa Representante de Equipamentos Pesados.

Revista Brasileira de Marketing, [S.l.], v. 14, n. 2, p. 86-99, 2015.

ASHLEY, C. et al. Why customers won't relate: Obstacles to relationship marketing engagement. Journal of

Business Research, [S.l.], v. 64, n. 7, p. 749-756, 2011. 
BALLANTYNE, D.; CHRISTOPHER, M.; PAYNE, A. Relationship marketing: looking back, looking forward. Marketing Theory, [S.l.], v. 3, n. 1, p. 159-166, 2003.

BALLANTYNE, D.; VAREY, R. J. Creating value-inuse through marketing interaction: the exchange logic of relating, communicating and knowing. Marketing Theory, [S.l.], v. 6, n. 3, p. 335-348, 2006.

BATTOR, M.; BATTOR, M. The impact of customer relationship management capability on innovation and performance advantages: testing a mediated model. Journal of Marketing Management, [S.l.], v. 26, n. 9-10, p. 842-857, 2010.

BERRY, L. L. Relationship marketing of services - growing interest, emerging perspectives. Journal of the Academy of Marketing Science, [S.l.], v. 23, n. 4, p. 236-245, 1995.

BERRY, L. L. Relationship Marketing. In: BERRY, L. L.; SHOSTACK, G. L.; UPAH, G. D. (Ed.) Emerging Perspectives on Service Marketing. American Marketing Association, Chicago, IL. 1983, p. 25-38.

BERRY, L. L.; GRESHAM, L. G. Relationship retailing: transforming customers into clients. Business Horizons, [S.l.], v. 29, n. 6, p. 43-47, 1986.

\section{BRETZKE, M. Marketing de Relacionamento e} competição em tempo real. São Paulo: Editora atlas, 2000.

COMIOTTO, F. D. et al. Comprei Meu Carro, o que vem depois? Análise da Percepção e da Importância Atribuídas por Clientes em Serviços de Pós-Venda. Revista

Brasileira de Marketing, [S.l.], v. 13, n. 6, p. 80-93, 2014.

CREMONEZI, G. et al. As Indústrias de Produtos Orgânicos, o Marketing de Relacionamento e o Endomarketing: Estudo de Múltiplos Casos. Revista Organizações em Contexto-online, [S.l.], v. 9, n. 17, p. 343-367, 2013.

D’ANGELO, A. C.; SCHNEIDER, H.; LARÁN, J. A. Marketing de relacionamento junto a consumidores finais: um estudo exploratório com grandes empresas brasileiras. Revista de Administração Contemporânea, [S.I.], v. 10, n. 1, p. 73-93, 2006.
DAS, K. Relationship marketing research (1994-2006): an academic literature review and classification. Marketing Intelligence \& Planning, [S.l.], v. 27, n. 3, p. 326-363, 2009.

DEMO, G.; BATELLI, L.; ALBUQUERQUE, P. Customer Relationship Management Scale for Video Games' Players: Exploratory and Ordinal Factor Analysis. Revista Organizações em Contexto, [S.l.], v. 11, n. 22, p. 285312, 2015.

DEMO, G.; PESSOA, R. CRM na administração pública: desenvolvimento e validação de uma Escala de Relacionamento com o Cidadão (ERCi). Revista de Administração Pública, [S.l.], v. 49, n. 3, p. 677-697, 2015.

DEMO, G.; ROZZETT, K. Customer Relationship Management Scale for the Business-to-Consumer market: exploratory and confirmatory validation and models comparison. International Business Research, [S.I.], v. 6, p. 29-42, 2013

DEMO, G. et al. Marketing de Relacionamento (CRM): Estado da Arte, Revisão Bibliométrica da Produção Nacional de Primeira Linha, Institucionalização da Pesquisa no Brasil e Agenda de Pesquisa. Revista de Administração Mackenzie, [S.l.], v. 16, n. 5, 2015.

DYBÅ, T.; DINGS $\varnothing Y R, T$. Streingth of evidence in Systematic Reviews in software engineering. Empirical Software Engineering and Measurement (ESEM), [S.l.], v. 8, 2008.

ERNST, H. et al. Customer relationship management and company performance-the mediating role of new product performance. Journal of the Academy of Marketing Science, [S.l.], v. 39, n. 2, p. 290-306, 2011.

FARIA, L. H. L. et al. 20 anos de publicações sobre marketing de relacionamento no Brasil: uma análise da produção acadêmica de 1992 a 2012 . Revista Brasileira de Marketing, [S.I.], v. 13, n. 1, p. 106-118, 2014.

FREITAS, W. R. S.; CUNICO, E.; PEDRON, C. D. Marketing de Relacionamento e Customer Relationship Management: uma análise da produção científica nacional à luz do componente" recursos humanos". Revista de Ciências da Administração, [S.l.], v. 17, n. 42, p. 9, 2015. 
FROW, P. E.; PAYNE, A. F. Customer relationship management: a strategic perspective. Journal of

Business Market Management, [S.l.], v. 3, n. 1, p. 7-27, 2009.

GRÖNROOS, C. Service management and marketing: customer management in service competition. [S.l.]: John Wiley \& Sons, 2007.

GRÖNROOS, C. Service logic revisited: who creates value? And who co-creates?. European Business

Review, [S.l.], v. 20, n. 4, p. 298-314, 2008.

GRÖNROOS, C. Marketing as promise management: regaining customer management for marketing. Journal of Business \& Industrial Marketing, [S.l.], v. 24, n. 5/6, p. 351-359, 2009.

HANSLIN, K.; RINDELL, A. Consumer-brand relationships in step-down line extensions of luxury and designer brands. Journal of Fashion Marketing and Management, [S.l.], v. 18, n. 2, p. 145-168, 2014.

HEALY, M. et al. The old, the new and the complicated-A trilogy of marketing relationships. European Journal of Marketing, [S.l.], v. 35, n. 1/2, p. 182-193, 2001.

HUANG, M. The influence of relationship marketing investments on customer gratitude in retailing. Journal of Business Research, [S.l.], v. 68, n. 6, p. 1318-1323, 2015.

IGLESIAS, O.; SAUQUET, A.; MONTAÑA, J. The role of corporate culture in relationship marketing, European Journal of Marketing, [S.I.], v. 45, n. 4, p. 631-650, 2011.

KASSARJIAN, H. H. Personality and consumer behavior: A review. Journal of Marketing Research, [S.l.], p. 409418, 1971.

KAUR, G.; SHARMA, R. D.; MAHAJAN, N. Exploring customer switching intentions through relationship marketing paradigm. International Journal of Bank Marketing, [S.I.], v. 30, n. 4, p. 280-302, 2012.

LEE, J.; KIM, S.; PAN, S. The role of relationship marketing investments in customer reciprocity. International Journal of Contemporary Hospitality Management, [S.I.], v. 26, n. 8, p. 1200-1224, 2014.
LIN, R.; CHEN, R.; CHIU, K. K. Customer relationship management and innovation capability: an empirical study. Industrial Management \& Data Systems, [S.I.], v. 110, n. 1, p. 111-133, 2010.

LING-YEE, L. Marketing metrics' usage: Its predictors and implications for customer relationship management. Industrial Marketing Management, [S.l.], v. 40, n. 1, p. 139-148, 2011.

LOURENÇO, C. D. S. Cultura Brasileira e Marketing de Relacionamento: Um Estudo Etnográfico no Varejo. Revista Brasileira de Marketing, [S.I.], v. 13, n. 5, p. 47-64, 2014

LOURENÇO, C. D. S.; SETTE, R. S. Relacionamentos de marketing em empresas varejistas: aspectos positivos e negativos. Revista Brasileira de Marketing, [S.l.], v. 12, n. 3, p. 152-178, 2013.

MAGALHÃES, M. R. A.; CRNKOVIC, L. H.; MORETTI, S. A. Importância do Marketing de relacionamento para a melhoria do atendimento e da vantagem competitiva nos serviços médicos privados. Revista Brasileira de Marketing, [S.l.], v. 8, n. 1, p. 70-92, 2010.

MENDES, V. L.; PEDRON, C. D. Programas de Fidelidade: um Estudo de Múltiplos Casos em Empresas Portuguesas. Revista Brasileira de Marketing, [S.I.], v. 13, n. 1, p. 60-74, 2014.

MILAN, G. S.; DE TONI, D. A Construção de um modelo sobre a retenção de clientes e seus antecedentes em um ambiente de serviços. REAd-Revista Eletrônica da Administração, [S.l.], v. 18, n. 2, p. 433-467, 2012.

MORGAN, R. M.; HUNT, S.D. The commitment-trust theory of relationship marketing. Journal of Marketing, [S.l.], v. 58, p. 20-38, 1994.

MOTA, M. O. Measuring moderations: a cross cultural and comparative research in services between brazilians and canadians. Review of International Business, [S.I.], v. 9, n. 2, p. 39-58, 2014.

O'MALLEY, L.; TYNAN, C. Relationship marketing in consumer markets - rhetoric or reality? European Journal of Marketing, [S.1.], v. 34, n. 7, p. 797-815, 2000. 
PAYNE, A. Handbook of CRM: achieving excellence in customer management. Oxford: Elsevier, 2006.

PEDRON, C. D.; SACCOL, A. Z. What lies behind the concept of customer relationship management? Discussing the essence of CRM thriugh a phenomenological approach. Brazilian Administration Review, v. 6, n. 1, p. 34-49, 2009.

PUPOVAC, L.; ZEHETNER, A.; SUDAREVIC, T. The ways of using CRM systems: the survey of literature.

Management Information Systems, [S.l.], v. 7, n. 2, p. 17-23, 2012.

RICHARDS, K. A.; JONES, E. Customer relationship management: Finding value drivers. Industrial

Marketing Management, [S.I.], v. 37, n. 2, p. 120-130, 2008.

ROCHA, L.; CLARO, D. P. Impacto do Marketing de Relacionamento sobre a Recomendação: Evidência do Varejo de Materiais de Construção Brasileiro. Revista Brasileira de Marketing, [S.I.], v. 12, n. 3, p. 123-151, 2013.

ROCHA, T. V. et al. Estudo Exploratório sobre o uso das Redes Sociais na Construção do Relacionamento com Clientes. Revista Brasileira de Gestão de Negócios, [S.l.], v. 15, n. 47, p. 262-282, 2013.

RYALS, Lynette. Making customer relationship management work: the measurement and profitable management of customer relationships. Journal of Marketing, [S.l.], v. 69, n. 4, p. 252-261, 2005.

SEMPREBON, E. et al. A importância da interdependência no relacionamento com marcas. REGE Revista de Gestão, [S.l.], v. 21, n. 4, p. 561-577, 2014.

SHETH, J. N.; PARVATIYAR, A. The evolution of relationship marketing. International Business Review, [S.l.], v. 4, n. 4, p. 397-418, 1995.

SILVA, L. A. et al. Marketing de Relacionamento em Diferentes Níveis de Contato em Redes Associativistas de Varejo. Revista Brasileira de Marketing, [S.I.], v. 11, n. 2, p. 178-202, 2012.
SOARES, G.; BORTOLUZZO, A. B.; BARROS, H.

$\mathrm{M}$. Determinants of the choice of marketing channels by corporate clients: an analysis of the information technology sector. Journal of Information Systems and Technology Management, [S.l.], v. 9, n. 3, p. 515-540, 2012.

SRINIVASAN, S. S.; ANDERSON, R.; PONNAVOLU, K. Customer loyalty in e-commerce: an exploration of its antecedents and consequences. Journal of Retailing, [S.l.], v. 78, n. 1, p. 41-50, 2002.

VAVRA, T. Marketing de relacionamento: como manter a fidelidade de clientes através do marketing de relacionamento. São Paulo: Atlas, 1993.

VARGO, S. L.; LUSCH, R. F. Service-dominant logic: continuing the evolution. Journal of the Academy of Marketing Science, [S.l.], v. 36, n. 1, p. 1-10, 2008.

WILSON, E. J; VLOSKY, R. P. Partnering relationship activities: building theory from case study research.

Journal of Business Research, [S.I.], v. 39 n. 1, p. 59$70,1997$.

WU, W.; TSAI, C.; FU, C. The Relationships among Internal Marketing, Job Satisfaction, Relationship Marketing, Customer Orientation, and Organizational Performance: An Empirical Study of TFT $\square$ LCD Companies in Taiwan. Human Factors and Ergonomics in Manufacturing \& Service Industries, [S.I.], v. 23, n. 5, p. 436-449, 2013.

ZABLAH, A. R.; BELLENGER, D. N.; JOHNSTON, W. J. An Evaluation of Divergent Perspectives on Customer Relationship Management: Towards a Common Understanding of an Emerging Phenomenon. Industrial Marketing Management, North Holland, v. 33, p. 475489, 2004. 